\title{
SURVIE DES ENFANTS ET PAUVRETÉ AU CONGO : APPLICATION D’UN MODÈLE DE DURÉE
}

\author{
(1) Samuel Ambapour, (2) Rufin Bidounga ${ }^{1}$, ${ }^{(1)}$ Stève Mboko Ibara \\ (1) Institut National de la statistique, Brazzaville, Congo \\ (2) Ecole Normale Supérieure, Université Marien Ngouabi, Brazzaville, Congo
}

Presented by Professor Dominique Mizère, member of the Corresponding Editors Board

RÉSumé. (French) La présente étude a pour principal objectif d'établir la causalité entre pauvreté et la mortalité des enfants de moins de cinq ans, à partir d'un modèle de durée. Dans cette recherche, les auteurs ont mis en évidence la pertinence du modèle de durée à risque proportionnel (modèle de Cox) pour analyser les déterminants de la mortalité des enfants de moins de cinq ans à travers l'âge au décès.

Les données utilisées proviennent de la première enquête démographique et de santé réalisée au Congo en 2005 . L'étude porte ainsi sur un échantillon de 4835 enfants nés vivants, parmi lesquels, 400 étaient décédés avant d'atteindre l'âge de 5 ans. La pauvreté est ici appréhendée dans l'optique non monétaire c'est-à-dire, à partir des caractéristiques des ménages en termes de bien d'équipements, de l'habitation et des conditions environnementales.

Les résultats empiriques mettent en évidence la forte contribution de la pauvreté dans l'explication de la mortalité infantile et infanto-juvénile. Au demeurant, le modèle de Cox ainsi estimé montre que les facteurs socio-économiques ont un impact significatif sur la mortalité des enfants de moins de cinq ans.

Asbtract (English) The main objective of this study is to establish causality between poverty and under five children's mortality, using a duration model. In this research, the authors highlighted the relevance of the proportional risk duration models (Cox regression) to analyze the determinants of the mortality of the children under five through the age at death. The data used come from the first demographic and health survey that was carried out inCongo in 2005 . The study thus carries on a sample of 4835 alive newborns, among whom, 400 had died before reaching the 5 years age. Poverty is apprehended here in nonmonetary frame i.e., using the characteristics of the households in terms of capital equipment, the dwelling and the environmental conditions. The empirical results highlight the strong contribution of poverty in the explanation of infant and under five mortality. Moreover, we showed, through the use of the Cox's model, that the socio-economic factors have a significant impact on the mortality of the children under five.

Keywords : Modèle de durée, fonction de survie, risque proportionnel, censure.

AMS 2010 Subject Classification : 62N01 - 91B15 - 97K80.

Copyright (C) 2015, African Journal of Applied Statistics. All rights reserved

Article history : Received 2015/08/27; Accepted 2015/12/15; Published Online 2015/12/31.

\section{INTRODUCTION}

Dans la littérature, on peut distinguer deux grandes catégories de modèles économétriques applicables à l'étude de la mortalité des enfants (Hala (2002)). Dans la première, on distingue des modèles du type logit (Aly et Grabowsky (1999), Mboko Ibara (2009)) ou probit (Melligton et Cameron (1999)). La variable dépendante dans ce cas est binaire (prenant la valeur 1 lorsque l'enfant est décédé, 0 dans le cas contraire). On peut cependant faire remarquer, qu'en donnant la même valeur à la variable dépendante sur un vaste intervalle, on perd une information précieuse (Ray (1988)). Dans le cas de la mortalité infantile (Ambapour (2001)), attribuer la valeur 1 aux cas de décès survenus dans l'année et la valeur 0 au-delà, c'est s'interdire de comprendre ce qui différencie les décès survenus au cours du premier mois de l'année (dite mortalité endogène) et ceux intervenus au cours du reste de cette période annuelle (dite mortalité exogène).

Dans ce papier, nous allons nous intéresser non pas au fait que l'enfant soit en vie ou décédé à un moment donné, mais plutôt à la transition d'un état à l'autre. Plus précisément, la modélisation concerne l'âge auquel l'enfant décède. Le problème ici c'est qu'il faut tenir compte du fait que pour certains enfants, on n'observe pas l'événement en question (le décès dans le cas qui nous intéresse). Ainsi, quel que soit le mode de collecte, prospectif ou rétrospectif, on est alors en présence de données

1. Corresponding author: (Rufin Bidounga) : rufbid@yahoo.fr Samuel Ambapour (ambapour_samuel@yahoo.fr),

Rufin Bidounga (rufbid@yahoo.fr),

Stève Mboko Ibara (stevemboko@yahoo.fr) 
incomplètes; l'information sur les trajectoires individuelles des enfants s'interrompt à la date de l'enquête et on ne connaît pas leur avenir. Le « récit » est interrompu. De telles durées sont dites censurées à droite (Lelièvre $(2010))^{2}$. Et, compte tenu de ce phénomène, on ne peut appliquer en toute rigueur les modèles de régression multiple car une des conditions de base n'est pas remplie (Ray (1988)) : l'absence d'erreurs de mesure sur la variable dépendante; d'où des estimateurs non efficaces, bien que non biaisés si le terme d'erreur est réellement aléatoire. Pour surmonter cette difficulté, nous utilisons une deuxième catégorie de modèles qui sont applicables lorsque l'on est en présence de données censurées. Ce sont des techniques économétriques spécialement conçues à cet effet : il s'agit des modèles de durée (hazard models) ou encore l'analyse des données de survie (survival data analysis) qui ont été pendant longtemps l'apanage des seuls démographes et actuaires et dont récemment les applications se sont étendues à d'autres domaines telles que la fiabilité, la biostatistique et l'économie. Cette modélisation est appliquée ici pour étudier la survie des enfants au Congo. En effet, bon nombre de travaux antérieurs ont adopté cette approche pour étudier la mortalité des enfants (Manda (199), Martin et al. (1983), Trussel (1983), Woldemicael (1998)).

Pour analyser l'âge auquel l'enfant décède, on utilise, les données de l'Enquête Démographique et de Santé réalisée en 2005 par le Centre National de la Statistique et des Etudes Economiques (CNSEE), avec l'assistance technique de ORC Macro, institution de coopération américaine en charge du programme international de ce type d'enquêtes (Demographic and Health Survey). De manière spécifique, cette enquête visait à atteindre un certain nombre d'objectifs dont notamment : recueillir des données à l'échelle nationale, représentatives selon le milieu de résidence, permettant de calculer divers indicateurs démographiques, en particulier les taux de fécondité, de mortalité infantile et juvénile; analyser les facteurs qui déterminent les niveaux et tendance de la fécondité et de la mortalité des enfants.

Selon cette enquête, pour la période allant de 2001 à 2005, le risque de mortalité infantile est évalué à 75 décès pour 1000 naissances vivantes; le risque de mortalité juvénile s'établit quant à lui à 44 pour mille. Globalement, le risque de mortalité infanto-juvénile, c'est-à-dire le risque de mortalité avant l'âge de cinq ans, est de $117 \%$. En fonction des données sur les variables explicatives, on a retenu un échantillon de 4.835 enfants nés vivants. Dans cet échantillon, on note 400 cas de décès, soit $8,27 \%$. C'est parmi les enfants de $0-1$ an que l'on observe le plus de cas de décès $(25,22 \%)$. Dans la période juvénile $(1-4$ ans), les décès représentent $2,52 \%$.

Cela étant, comme les niveaux, tendances et caractéristiques de la mortalité des enfants sont fonction des conditions sanitaires, environnementales, socioéconomiques et culturelles qui prévalent dans une population et dans ces diverses couches sociales (CNSEE \& ORC Macro (2005)), l'objectif de ce papier est finalement, de tester l'influence de certains de ces facteurs en recourant aux méthodes statistiques d'analyse des durées.
Notre texte s'articule de la manière suivante : dans un premier temps, nous présenterons d'une manière générale, les modèles de durée ${ }^{3}$. L'accent sera d'abord mis sur les fonctions de survie, ensuite sur une famille de lois de probabilité utilisée pour modéliser la survie et enfin sur les modèles de durée conditionnels si l'on souhaite savoir quelles sont les variables qui influent sur les formes de fonctions de survie; dans un deuxième temps, nous étudierons les déterminants de la mortalité des enfants au Congo. Nous commencerons par la définition des variables de l'étude, suivra la présentation du modèle économétrique de durée retenu et enfin nous commenterons les résultats des estimations de ce modèle.

\section{Modèles De DuRÉE}

On peut noter quatre spécificités dans l'analyse des modèles de durée (Drosbecke, Fichet et Tassi (1989), Planchet and Thérond (2006)). La caractéristique fondamentale de ces données de durée est qu'elles sont constituées de variables aléatoires positives. Du point de vue des applications, ces variables représentent soit la durée passée dans un état donné (durée de vie d'un cancéreux par exemple), soit la durée séparant deux événements (durée séparant la naissance et la mort d'un enfant).

La deuxième particularité est que, le calcul de probabilité identifie la loi de probabilité de ces variables aléatoires par une fonction de répartition, une densité ou une fonction caractéristique; ce qui permet de définir d'autres notions associées, telles que la fonction de survie, la fonction de hasard, le taux de hasard cumulé.

Une troisième différence par rapport au modèle d'échantillonnage classique de la statistique est la présence de données incomplètes (modèle tronqué ou modèle censuré).

La quatrième particularité des données de durée est la présence des variables exogènes, qui ont un caractère essentiellement descriptif, et qui interviennent dans l'écriture de la loi de durée. Une distinction est alors faite selon la dépendance, ou non, de ces variables par rapport au temps.

Dans ce qui va suivre, nous allons tenter d'expliciter certaines de ces particularités, d'autres ayant déjà été évoquées en introduction ${ }^{4}$.

2.1. Définitions des fonctions de survie. Dans une étude de survie des enfants, il faut connaître pour chacun d'eux la date du début de l'observation qu'on appelle date d'origine (date de naissance) et la date de dernières nouvelles (date de décès). La durée de vie $T$ des enfants est donc le délai écoulé entre ces deux dates. On considère que $T$ est une variable aléatoire de loi continue et à valeurs dans $[0, \infty[$.

On note $f(t)$ la fonction de densité de $T$ et l'on a :

$$
f(t)=\frac{\mathrm{d}}{\mathrm{d} t} F(t)=\lim _{\mathrm{d} t \rightarrow 0} \frac{\operatorname{Pr}(t \leq T \leq \mathrm{d} t+t)}{\mathrm{d} t}
$$

La fonction de répartition $F(t)$ de la variable $T$, est la probabilité de décéder entre 0 et $t$

$$
F(t)=\operatorname{Pr}(t \leq T)=\int_{0}^{t} f(u) \mathrm{d} u
$$

2. Concernant la censure à gauche, elle se produit dès que l'on dispose d'informations sur l'état des personnes à un moment donné sans disposer de la durée depuis laquelle elles sont dans cet état (Lelièvre (2010)).

3. Sur l'analyse statistique des durées de vie, on pourra consulter les ouvrages suivants : Gourieroux (1989), Courgeau et Lelièvre (2014), Kalbfleisch et Prentice (1989), Planchet and Thérond (2006), ainsi que la synthèse de Kiefer (1988) et l'article de vulgarisation de Ray (1988).

4. Cas de la censure. 
On s'intéresse généralement à la probabilité de survivre audelà de $t$ que l'on nomme fonction de survie. On a :

$$
S(t)=1-F(t)=1-\int_{\infty}^{t} f(u) \mathrm{d} u=1-\int_{0}^{t} f(u) \mathrm{d} u
$$

Avec $S\left(0_{+}\right)$et $\lim _{t \rightarrow \infty} S(t)=0$.

On définit le risque instantané de décès, ou plus correctement le taux instantané de décès ${ }^{5}$, par la fonction :

$$
\lambda(t)=\lim _{\mathrm{d} t \rightarrow 0} \frac{\operatorname{Pr}(t \leq T \leq \mathrm{d} t+t \mid T>t)}{\mathrm{d} t}
$$

Soit

$$
\lambda(t)=\frac{f(t)}{S(t)}=-\frac{\mathrm{d}[\ln S(t)]}{\mathrm{d} t}
$$

$\lambda(t)$ est la probabilité de décéder entre $t$ et $t+\mathrm{d} t$ pour un enfant, conditionnellement au fait que cet enfant est encore vivant en t. $\lambda(t)$ est encore appelé force de la mortalité ou fonction de risque ou encore fonction de hasard. On a donc :

$$
S(t)=\exp \left[-\int_{0}^{t} \lambda(u) \mathrm{d} u\right], t \in \mathbb{R}_{+}
$$

Enfin, on définit $\Lambda(t)$ comme la fonction de risque cumulée de $\lambda(u)$ entre 0 et $t:$

$$
\Lambda(t)=\int_{0}^{t} \lambda(u) \mathrm{d} u
$$

pour laquelle

$$
S(t)=e^{-\Lambda(t)}
$$

de sorte que

$$
\Lambda(t)=-\ln [S(t)]
$$

et

$$
f(t)=\lambda(t) \exp [-\Lambda(t)]
$$

La façon dont la fonction hasard dépend du temps est souvent très importante au niveau de l'interprétation. Dans le cas de la mortalité, on peut distinguer trois cas (Hill et al. (1996)) :

- $\lambda(t)$ constant, le risque instantané de décès ne dépend pas du temps. On dit qu'il n'y a pas vieillissement;

- $\lambda(t)$ est une fonction croissante : le risque de décès augmente avec l'âge. On dit qu'il y a vieillissement;

- $\lambda(t)$ est une fonction décroissante. C'est le cas du risque instantané de décès pour les enfants de moins de un an qui diminue avec l'âge entre zéro et un an.

La force de la mortalité d'une population humaine dans son ensemble a une forme de $\mathrm{U}$ : forte à la naissance, décroissante, puis croissante.

2.2. Quelques modèles paramétriques de survie. Dans la pratique plusieurs familles de loi de probabilité sont utilisées pour modéliser les durées. Signalons les plus employées ${ }^{6}$ (Gourieroux (1989), Kiefer (1988)) :

2.2.1. Loi exponentielle. $T$ suit une loi exponentielle de paramètre $\gamma$ si :

$$
\begin{gathered}
F(t)=1-\exp (-\gamma t) \\
S(t)=\exp (-\gamma t) \\
f(t)=\gamma \exp (-\gamma t) \\
\lambda(t)=\gamma ; t>0 .
\end{gathered}
$$

5. Qu'on appelle dans certains cas "taux de rupture" (failure rate)

6. On peut citer aussi la loi log-normale et la loi gamma.
On a $\ln S(t)=\gamma t \Rightarrow$ la courbe $(t,-\ln \hat{S}(t))$ est approximativement une droite.

2.2.2. Loi de Weibull. C'est une famille de lois indexée par deux paramètres généralisant la loi exponentielle. $T$ suit une loi de Weibull de paramètre de paramètre $\gamma>0$ et $\alpha>0$ si :

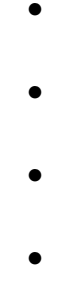

Nous avons

$$
\begin{gathered}
F(t)=1-\exp \left(-\gamma t^{\alpha}\right) \\
S(t)=\exp \left(-\gamma t^{\alpha}\right) \\
f(t)=\gamma \alpha t^{\alpha-1} \exp \left(-\gamma t^{\alpha}\right) \\
\lambda(t)=\gamma \alpha t^{\alpha-1},
\end{gathered}
$$

Dès lors, la fonction de hasard de Weibull est monotone croissante ou décroissante selon que $\alpha>0$ ou $\alpha<0$;

$\ln (-\ln S(t))=\ln \gamma+\alpha \ln t \Rightarrow$ la courbe $[\ln t, \log (-\ln \hat{S}(t))]$ est approximativement une droite.

2.2.3. Loi log-logistique. $T$ suit une loi log-logistique de paramètre $\gamma>0$ et $\alpha>0$ si :

$$
\begin{gathered}
F(t)=1-\left[\frac{1}{1+(\gamma t)^{\alpha}}\right] \\
S(t)=\frac{1}{1+(\gamma t)^{\alpha}} \\
\qquad(t)=\frac{\gamma \alpha(\gamma t)^{\alpha-1}}{\left(1+\gamma t^{\alpha}\right)^{2}} \\
\qquad \begin{array}{l}
\text { • } \\
\text { • }
\end{array} \\
\text { On a : } \\
\lambda(t) \text { monotone décroissante si } \gamma<1 \text { et } \lim _{t \rightarrow 0} \lambda(t)=\gamma \\
\lambda(t) \text { monotone croissante si } \gamma>1 \text { et } \lim _{t \rightarrow 0} \lambda(t)=\infty \\
\lambda(t) \text { est croissante puis décroissante } \operatorname{si} \gamma>1 \text { avec } \lim _{t \rightarrow 0} \lambda(t)=0 \\
\text { et } \lim _{t \rightarrow \infty} \lambda(t)=0 .
\end{gathered}
$$

2.3. Modèles de durée conditionnels. Ici, on souhaite savoir quelles sont les variables qui influent sur les formes de fonctions de hasard introduites ci-haut. Pour ce faire, il est nécessaire de faire dépendre la loi de durée des valeurs de ces variables. On retient, dans ce texte les variables explicatives individuelles; celles qui ne sont pas des fonctions du temps. Ces variables explicatives $x$ sont introduites dans la loi, avec un paramétrage $\beta$. La fonction de hasard relative à l'individu $i$ s'écrit :

$$
\lambda_{i}(t)=\lambda\left(x_{i}, t, \beta\right)
$$

On distingue deux types de modèle, les modèles à risque proportionnel et les modèles à risque accéléré. 
2.3.1. Les modèles à risque proportionnel. Dans cette classe de modèles, $x$ est sensé avoir un effet multiplicatif sur la fonction de hasard.

On a :

$$
\lambda\left(t, x, \beta, \lambda_{0}\right)=\phi(x, \beta) \lambda_{0}(t),
$$

où $\lambda_{0}$ est le hasard de base correspondant à $\phi(\cdot)=1$. Une spécification de $\phi$ généralement utilisée est :

$$
\phi(x, \beta)=\exp \left(x^{\prime} \beta\right)
$$

2.3.2. Les modèles à risque accéléré. Dans ce cas, $x$ a un effet multiplicatif sur la durée elle-même ou encore un effet additif sur son logarithme. La fonction de hasard est donnée par :

$$
\lambda(t, x, \beta)=\lambda_{0}[t \phi(x, \beta)] \phi(x, \beta)
$$

Dans le cas spécifique et important où $\phi(x, \beta)=\exp \left(x^{\prime} \beta\right)$, le modèle à risque accéléré peut être représenté sous une forme log-linéaire, si l'on fait le changement de variable adéquat (Kiefer (1988)). En effet, soit par exemple $T_{0}$ une variable aléatoire représentant l'âge au moment du décès pour un enfant issu d'une population homogène dont les caractéristiques correspondent aux valeurs nulles des variables explicatives. On peut retenir le modèle suivant :

$$
T=\exp \left(a+x^{\prime} \beta\right) T_{0},
$$

où, $a$ représente le terme constant du modèle à estimer ;finalement, on peut écrire (2.24) sous la forme log-linéaire suivante :

$$
\ln T=a+x^{\prime} \beta+\ln T_{0}+\varepsilon
$$

\section{LES DÉTERMINANTS DE LA MORTALITÉ DES ENFANTS}

Dans cette deuxième partie du texte, nous présenterons le modèle de durée retenu pour analyser l'âge auquel l'enfant décède. Nous définirons ensuite les variables de l'étude. Enfin nous commenterons les résultats de l'estimation de notre modèle. Ces résultats seront précédés d'une analyse sommaire de la fonction de survie des enfants selon une approche non paramétrique.

3.1. Le modèle empirique. Nous retenons dans ce papier un modèle, sans doute le plus utilisé en sciences sociales (Le Golf (2003)), celui de $\operatorname{Cox}^{7}$ (Cox (1972), Cox (1975)). Il correspond à la spécification de type (2.21), c'est-à-dire des modèles à risque proportionnel. Dans cette fonction, on considère le hasard de base $\lambda_{0}$ comme un paramètre de nuisance à éliminer. Cependant, comme $\lambda_{0}$ est non défini, le modèle est partiellement non spécifié, et par conséquent appelé semi-paramétrique. Notons par (Gourieroux,1989) :

- $Y_{(i)} \leq \ldots \leq Y_{(n)}$, les observations ordonnées des diverses variables ;

- $C_{(i)}$ la variable indiquant si l'observation $y_{(i)}$ est ou non complète ;

. et $\mathfrak{R}\left(Y_{(i)}\right)=\left\{j: Y_{(j)} \geq Y_{(i)}\right\}$ l'ensemble des individus encore présents dans la population juste avant que celui associé à $Y_{(i)}$ ne disparaisse. Sachant ceux encore présents, la probabilité que celui qui disparaît soit justement associé à $Y_{(i)}$ est :

$$
\frac{\lambda\left(Y_{(i)} ; x, \beta, \lambda_{0}\right)}{\sum_{j \in \mathfrak{R}\left(Y_{(j)}\right)} \lambda\left(Y_{(j)} ; x, \beta, \lambda_{0}\right)}
$$

Cette expression se réduit à :

$$
\frac{\exp \left(x_{(i)} \beta\right)}{\sum_{j \in \mathfrak{R}\left(Y_{(j)}\right)} \exp \left(x_{j} \beta\right)^{\prime}},
$$

où $x_{(i)}$ désigne la valeur des variables explicatives associées à $Y_{(i)}$. La vraisemblance ${ }^{8}$ partielle de Cox est alors formée en faisant le produit sur les observations complètes de ces probabilités conditionnelles :

$$
\ell_{p}(\beta)=\prod_{i=1}^{n}\left[\frac{\exp \left(x_{(i)} \beta\right)}{\sum_{j \in \mathfrak{R}\left(Y_{(j)}\right)} \exp \left(x_{j} \beta\right)}\right]^{c_{i}}
$$

L'estimateur du maximum de vraisemblance partielle de Cox est alors défini comme solution $\hat{\beta}$ du problème

$$
\max _{\beta} \ln \ell_{p}(\beta)=\sum_{i=1}^{n} c_{i} \ln \left[\frac{\exp \left(x_{(i)} \beta\right)}{\sum_{j \in \mathfrak{R}\left(Y_{(j)}\right)} \exp \left(x_{j} \beta\right)}\right]
$$

$\hat{\beta}$ est convergent, asymptotiquement normal et admet une précision asymptotique pouvant être approchée par :

$$
-\frac{\partial^{2} \ell_{n} l_{p}(\beta)}{\partial \beta \partial \beta^{\prime}}
$$

3.2. Définition des variables. On va spécifier successivement :

- la variable dépendante;

- les variables explicatives.

3.2.1. La variable dépendante. La variable dépendante de notre modèle est l'âge au décès de l'enfant. Cet âge est soit observé, soit censuré. Dans le premier cas, il s'agit du temps (en mois) vécu de la naissance au décès. Dans le deuxième, il s'agit de l'âge à la date de l'enquête que l'on introduit dans le modèle, une seconde variable, la variable indicatrice ${ }^{9}$ indiquant alors s'il y a ou non censure à droite

$$
c_{i}= \begin{cases}1 & \text { n'y a pas censure } \\ 0 & \text { sinon }\end{cases}
$$

3.2.2. Les variables explicatives. Plusieurs théories et schémas explicatifs de la mortalité des enfants ont été proposés dans la littérature (Masuy-Stroobant (2002)). Cependant, le modèle de Mosley et Chen (1984) est sans doute, celui qui a reçu le plus d'écho chez les chercheurs ${ }^{10}$ (Agha (2000) Beaulière, Flori et Boidin (2007), Sastry (1996)). Ces deux auteurs ont proposé un schéma qui intègre l'approche des sciences sociales et celle des sciences bio-médicales. Leur approche fait donc la distinction entre les variables indépendantes (ou sous-jacentes ou socio-économiques) et les variables dépendantes (biomédicales ou intermédiaires ou déterminants proches).

7. En effet, l'utilisation de ce modèle dans de nombreux types d'études a fait que son usage s'est beaucoup répandu ces dernières années pour analyser des données censurées.

8. Il ne s'agit pas vraiment d'une vraisemblance au sens usuel, mais plutôt d'une vraisemblance marginale (Drosbecke, Fichet et Tassi (1989); Kalbfleisch et Prentice (1989)).

9. Comme on peut le constater, la présence de la censure oblige à dédoubler la variable dépendante.

10. Ce modèle est souvent retenu pour trois raisons (Beaulière, Flori et Boidin (2007)) : premièrement, il est le plus fréquemment cité dans la littérature traitant du sujet; deuxièmement, il essaie d'intégrer les méthodes de recherche utilisées par les chercheurs en sciences sociale et médicale; troisièmement, il est étroitement lié à la théorie de Cadwell sur le rôle de l'éducation de la mère dans la détermination de la santé des enfants. 
Les effets des variables indépendantes étant considérés comme indirects parce que agissant à travers les variables biomédicales. Dans ce schéma, les variables socio-économiques sont regroupées en trois catégories : variables individuelles, variables relatives au ménage et variables communautaires. Par ailleurs ils ont identifié 14 déterminants proches, classés en cinq groupes : facteurs maternels, contamination de l'environnement, disponibilité alimentaire, lésions et blessures et contrôle individuel de la santé et de la maladie.

Comme indiqué en introduction, pour modéliser l'âge au décès, nous utilisons les données de l'enquête démographique et de santé réalisée par le CNSEE en 2005. Les limites de ce type de données sont de deux ordres. Premièrement, ce sont des données qui proviennent d'une enquête transversale rétrospecpective qui font appel à la mémoire. Cette information peut donc être considérée comme fragile. Deuxièmement, si l'enquête apporte une information rétrospective sur le statut de l'enfant, on ignore en revanche quelle était par exemple la situation socio-économique des parents lors des différentes étapes de l'itinéraire de l'enfant.Pour modéliser l'âge au décès de l'enfant nous adoptons donc une hypothèse forte, celle qui consiste à étudier le lien entre cet âge et les principaux évènements de la vie familiale et professionnelle des parents en référence à la situation actuelle (ou date de l'enquête).

Suivant le schéma explicatif proposé par Mosley et Chen (1984) et par rapport aux données disponibles, on peut classer les variables explicatives en deux catégories (Madise, Banda et Benaya (2003), Mutunga (2007)). Dans le premier groupe, les variables socio-économiques suivantes ont été retenues :

- la pauvreté;

- le niveau d'instruction de la mère;

- les matériaux du sol ;

- la disponibilité de l'eau et de l'électricité.

Les deux premières variables ${ }^{11}$ sont fréquemment utilisées comme facteurs explicatifs de la mortalité dans les pays en développement (Casterlin, Cooksey et Ismail (1989)). En ce qui concerne le revenu, on ne peut pas nier que l'aisance économique est un facteur évident d'accès aux moyens de la santé. Nous ne disposons pas malheureusement des données sur le revenu ou les consommations des ménages; on a construit à la place un indice de richesse à partir des informations relatives aux biens durables possédés par les ménages. Cet indice est utilisé comme un proxy du revenu de long terme des ménages. Ce dernier a été ensuite décomposé en trois classes socio-économiques : pauvre, moyenne et riche. On s'attend à ce que le revenu des ménages augmente les chances de survie de l'enfant.

Dans les pays en développement, l'instruction des mères est souvent retenue comme l'un des facteurs primordiaux de la baisse de la mortalité infantile ${ }^{12}$ (Caldwell (1979)). Beaucoup de travaux empiriques ont d'ailleurs confirmé l'existence d'une telle relation(Franzen et Hogan (1982), Schultz (1984), Aly et Grabowsky (1999), Melligton et Cameron (1999)). De leur côté, Tabutin et Akoto (cité par Caselli, Vallin et Wunsh
(2002)) ont indiqué qu'en Afrique subsaharienne, une telle relation négative existe bien mais que l'intensité de cette relation varie beaucoup selon les pays et qu'elle est nettement plus forte pour la mortalité entre un et cinq ans qu'avant un an.

Les trois variables suivantes, l'eau, l'électricité, les matériaux de sol sont souvent également utilisées comme indicateurs de niveau de vie et sont souvent incluses dans la construction de l'indice de richesse. Cependant, la littérature antérieure exige parfois de les traiter séparément, parce qu'elles sont liées à la fois aux équipements collectifs et au patrimoine du ménage. Nombreuses sont les études qui ont mis en lumière l'importance de ces infrastructures pour les pathologies infantiles ${ }^{13}$. Précisément, en ce qui concerne l'eau potable, une abondante littérature existe. A cet effet, on peut citer les travaux de Esrey (1996) sur un panel de pays, Woldemicael $(1998)$ ) en ce qui concerne l'Erythrée, Hala (2002) pour l'Egypte, Jacoby et Wang (2003) pour la Chine. A ce sujet, Woldemicael (1998) note que les conditions d'environnement des ménages et particulièrement l'approvisionnement en eau semble avoir joué un rôle majeur dans la baisse de la mortalité dans certaines villes des Etats-Unis et en Europe au XIXe siècle et, plusieurs études dans les zones urbaines des pays en développement ont démontré l'existence de cette étroite liaison.

Le deuxième groupe comprend essentiellement les variables démographiques ${ }^{14}$ :

- l'âge de la mère à l'accouchement ;

- le rang de naissance;

- le type de naissance;

- le nombre d'enfants de $0-4$ ans dans le ménage;

- le poids de l'enfant à la naissance.

En ce qui concerne l'âge de la mère à l'accouchement, on pourrait penser qu'une grossesse à un âge avancé risque de conduire à des malformations congénitales liées au vieillissement de la femme. Les enfants des femmes âgées risquent ainsi d'être plus vunérables ${ }^{15}$.

Le rang de naissance joue un rôle crucial au niveau de la survie de l'enfant. Il a été suggéré que le rang des naissances semble avoir un effet significatif sur la qualité de vie, y compris sur la mortalité infantile. Contrairement aux premiers nés, les enfants de rang élevé bénéficient généralement de soins de moindre qualité, l'attention accordée par la mère aux enfants diminuant considérablement avec le rang de naissance de l'enfant. Cette diminution provient du surcroît de charge occasionné par une famille relativement nombreuse. Par contre, les enfants des premiers rangs sont généralement issus d'un accouchement à un âge précoce et sont donc exposés à une insuffisance pondérale ou une prématurité. Par ailleurs, la présence d'un nombre croissant d'enfants de moins de cinq ans dans le ménage augmente la charge de la mère en matière de soins et par conséquent pourrait avoir un impact sur la survie des enfants.

11. Le revenu et le niveau d'éducation sont reconnus par la Banque Mondiale et l'Organisation Mondiale de la Santé comme d'excellents déterminants de la santé des individus.

12. Caldwell (1979) a établi le fait que le niveau d'instruction, plus spécifiquement celui de la mère est la caractéristique socio-économique la plus discriminante en matière de survie des enfants.

13. Par exemple, l'accès à l'eau potable et l'existence d'un sol en ciment évitent de nombreuses maladies, notamment intestinales.

14. Une variable importante n'a pas été prise au compte parce que présentant un fort taux de non réponse. Il s'agit de l'intervalle inter génésique. En effet, des intervalles inter génésiques très courts sont un facteur déterminant de la mortalité des enfants.

15. S'agissant de l'âge de la mère au moment de l'accouchement, il faut mentionner également que les âges précoces constituent aussi un facteur d'augmentation du risque de mortalité infanto-juvénile. 
S'agissant du type de naissance, Morrison et Linskens (cité par Ambapour et Moussana Hylod (2008)) notent que chaque jumeau souffre souvent à la naissance d'un handicap qu'il faudrait compenser par une alimentation et des soins adaptés.

Pour les pédiatres, le poids de naissance est un indicateur de fragilité de l'enfant. D'après McCormick (cité par MasuyStroobant (2002)), une étroite association s'établit de façon systématique entre le poids de naissance et le risque de décès, $\mathrm{y}$ compris dans les pays en voie de développement. Selon cet auteur, le poids à la naissance est probablement le facteur unique le plus important de la mortalité néonatale, sans compter qu'il est un déterminant significatif de la mortalité post-néonatale ainsi que de la mortalité infantile et juvénile.

3.3. Résultats des estimations. Nous allons décrire nos estimations et les résultats produits. Nous les commenterons amplement par rapport aux objectifs visés. Nous utilisons la figure FIG.1 et le tableau TAB.1 (Voir Appendice) résumant les estimations fournies par le modèle de Cox. Ces éléments sont donnés dans l'Appendice en fin de document.

Nous commençons par donner de façon sommaire les résultats obtenus par l'estimateur de Kaplan-Meier; suivront les estimations calculées selon le modèle de Cox.

3.3.1. Estimation non paramétrique de Kaplan-Meier. Cette méthode ${ }^{16}$ consiste à estimer la distribution de la fonction de survie , c'est-à-dire la distribution au cours du temps de la probabilité de ne pas avoir connu l'évènement auquel on s'intéresse. Considérons le temps de manière discrète et notons par(Le Golf (2003), Lelièvre (2010)) :

- $t_{i}$ un instant au cours duquel il y a l'observation d'au moins un évènement;

- $d_{i}$ et $c_{i}$, les effectifs des individus qui, respectivement, connaissent l'évènement et sortent d'observation (censures à droite) en $t_{i}$;

- $N_{i}$ la population soumise au risque en $t$.

La probabilité de survie en $t_{i}$ est alors :

$$
S\left(t_{i}\right)=\left(1-h_{i}\right) S\left(t_{i-1}\right)
$$

avec

$$
h_{i}=\frac{d_{i}}{N_{i}}
$$

On obtient alors $S(t)$ comme le produit de toutes les probabilités de n'avoir pas connu l'évènement depuis le début de l'observation :

$$
S(t)=\prod_{t_{i} \leq t}\left(1-h_{i}\right)
$$

Ainsi, l'étude descriptive de la survenue des décès selon l'âge peut être raffinée en tenant compte de la vitesse d'occurrence des décès (la cinétique des évènements); c'est ce que permet l'analyse de la fonction de survie par la méthode de KaplanMeier. Sur le graphique ci-dessous chaque plateau de l'escalier représente la probabilité de survie pendant l'intervalle de temps correspondant sur l'axe des abscisses. La hauteur de l'escalier représente la chute de la probabilité due aux décès constatés à l'instant.
Les plus grandes chutes de probabilité de survie s'observant à partir de 24 mois. Cette date correspond à l'âge de deux ans, soit l'âge modal au décès sur la période juvénile. Avant cette date, la fonction de survie est caractérisée par une plus ou moins régulière évolution; exceptée la chute rapide observée au cours du premier mois de vie. Cette allure dénote d'une certaine uniformité dans la répartition des décès avant le deuxième anniversaire. Au-delà, la probabilité de survie ne diminue que lentement.

On conclut à l'existence de deux phases dans l'évolution des enfants de moins de cinq ans selon l'âge : une baisse régulière et rapide de la probabilité de survie avant l'âge de deux ans, et de là commence une phase de lente baisse jusqu'au cinquième anniversaire. La rapide baisse initiale de la fonction de survie serait due aux décès néonataux.

3.3.2. Le modèle de Cox. Interprétation des coefficients estimés. On donne les résultats obtenus par le logiciel SPSS. Dans TABLE 1 (Voir Appendice), ce ne sont pas les valeurs des coefficient $\beta$ qui sont consignées, mais plutot l'exponentiel du coefficient donné sous $\exp (\beta)$; ce qui correspond à ce que l'on dénomme hazard ratio (Ritschard (2004)). Par ailleurs, l'estimation du modèle de est conditionnée par l'hypothèse de proportionnalité des risques ${ }^{17}$. Cette hypothèse a été testée en utilisant les procédures stphplot et stocoxkm du logiciel STATA. Ainsi, suivant Hala (2002), le modèle de Cox est estimé pour les périodes infantile (modèle $M_{1}$ ), juvénile (modèle $M_{2}$ et infanto-juvénile (modèle $M_{3}$ ).

3.4. (i) Mortalité infantile. De façon globale, le modèle est très satisfaisant avec un khi-deux statistiquement significatif au seuil de 1\%. En dehors des matériaux du sol, toutes les autres variables du modèle sont significatives. Toutes choses égales par ailleurs, le niveau de vie des ménages influence significativement la mortalité des enfants de $0-11$ mois. En effet, comparativement aux enfants des ménages pauvres, les enfants des ménages riches ont $40 \%$ plus de chance de ne pas décéder entre $0-11$ mois.

Au seuil de 5\%, le niveau d'instruction de la mère est significativement lié à l'âge au décès de l'enfant. Les enfants issus des mères de niveau d'instruction supérieur, ont $40 \%$ moins de risque de décéder que ceux des mères sans instruction. Ceux des mères de niveau primaire courent $50 \%$ de risque de décéder avant le premier anniversaire.

La disponibilité de l'eau/électricité concourt aussi à l'explication de la mortalité infantile au Congo. Contrairement à ce que l'on aurait pu s'attendre, le risque de mortalité des enfants est de $30 \%$ à $40 \%$ plus grand quand le ménage dispose d'un des deux éléments que lorsqu'il n'en dispose pas du tout. Les enfants des ménages disposant de l'eau et de l'électricité ont $20 \%$ de chances de survivre jusqu'à leur premier anniversaire que leurs homologues des ménages sans eau ni électricité.

Selon le rang de naissance, les enfants des premiers rangs courent $49 \%$ moins de risque de décès que ceux de rang cinq ou plus.

16. L'estimation de Kaplan et Meier (dite aussi produit limite (PL)) repose sur l'idée intuitive suivante : être encore en vie après l'instant t, c'est être en vie juste avant t et ne pas mourir à l'instant t (Hill et al. (1996)). L'avantage de cette méthode réside dans le fait qu'il ne nécessite pas de postuler une loi de probabilité spécifique pour les durées.

17. Cette hypothèse doit être grossièrement vérifiée pour pouvoir entreprendre l'analyse. On veut que $\ell_{n}[\lambda(t, x, \beta)] \approx \ell_{n}\left[\lambda_{0}(t)\right]+x^{\prime} \beta$ : l'écart entre les courbes est constant; les différentes courbes $\ell_{n}\left[\ell_{n} S(t)=\ell_{n}\left(\lambda_{0}(t)\right)\right]$, selon $\ell_{n}(t)$, à paramétrage fixé, sont parallèles entre elles. 
Plus le rang augmente, plus les chances de survie infantile s'amenuisent mais pas de façon significative.

Les naissances survenant quand la mère a un âge compris entre $15-19$ ans portent plus de préjudice aux enfants que celles survenant à partir de 20 ans.

En ce qui concerne les naissances multiples, elles sont associées à un risque de décès infantile de $67 \%$ fois plus important que les naissances simples.

Enfin, le poids à la naissance nous renseigne que les enfants naissant avec un faible poids courent $40 \%$ fois plus de risque de décès que ceux de poids normal et $60 \%$ de plus de risque que ceux en surpoids.

3.5. (ii) Mortalité juvénile. Le tableau TAB.1 (Voir Appendice) fournit également les résultats des estimations sur la période $1-4$ ans révolus. Dans cette période juvénile, le niveau de vie, le niveau d'instruction, la disponibilité en eau/électricité et l'âge de la mère à l'accouchement n'ont plus d'effets significatifs sur l'âge au decès.

Seules cinq variables sont significativement liées à l'âge au décès. Il s'agit du materiau du sol, du rang de naissance, du nombre d'enfants de moins de cinq ans dans le ménage, du type de naissance et du poids de l'enfant à la naissance.

En ce qui concerne le materiau du sol, les résultats montrent que les enfants vivant dans des maisons avec un sol en careaux ont plus de $80 \%$ de chances de survivre jusqu'au cinqième anniverssaire que leurs homologues vivant dans des maisons de sol en terre nue. Les différences sont non entre les enfants vivant dans des ménages de sol en ciment et ceux de la modalité de référence. Par rapport au rang de naissance, les premières naissances, qui interviennent généralement autour de 20 ans ${ }^{18}$ , ont statistiquement plus de $65 \%$ de chances de survivre que les naissances de rang cinq ou plus.

Les enfants issus des naissances simples ont plus de $60 \%$ de chances de survivre entre $1-4$ ans revolus que ceux des naissances multiples.

Le nombre d'enfants de moins de cinq ans dans le ménage agit négativement sur l'âge au décès. Plus il est élevé plus les enfants courent des risques importants de décéder dans l'intervalle d'âge $1-4$ ans révolus.

Selon le poids à la naissance, les enfants nés avec $2500 \mathrm{~g}$ ou plus (poids nornmal et surpoids) ont environ $56 \%$ de chances de plus de survivre au dela de 4 ans que ceux nés avec un faible poids.

3.6. (iii) Mortalité infanto-juvénile. Ce modèle est globalement significatif : toutes les variables explicatives se sont averées significatives, mais à de degrés de significativité divers.

La principale variable d'analyse, le niveau de vie des ménages est significative au seuil de $5 \%$. Même si le risque de décès des enfants des ménages moyens sont presque identiques à ceux des ménages pauvres ${ }^{19}$, les enfants des ménages riches ont $36 \%$ moins de risque de mourir que ceux des ménages pauvres. Toutefois, il convient de noter, toutes choses égales par ailleurs, que la pauvreté agit plus sur l'âge au décès des enfants de moins d'un an que sur celui des enfants de $1-4$ ans révolus.

Selon le niveau d'instruction, les risques propotionnels ne sont significatifs que pour les niveaux sécondaire et supérieur. Cela dit, les enfants des femmes sans instruction courent $25 \%$ plus de risque de décès que ceux des mères de niveau secondaire et $50 \%$ plus de risque de décès que ceux des mères de niveau supérieur. Ce résultat révèle la nécessité d'éduquer les femmes, car avec l'acquisition d'une certaine instruction, elles acquièrent également une meilleure connaissance des règles d'hygiène et plus généralement des principes de base en matière de surveillance de la petite enfance.

Les matériaux du sol des maisons sont un facteur à grand risque de décès pour les enfants de moins de cinq ans. En effet, comparés aux enfants vivant dans des maisons de sol en carreaux, les enfants des maisons de sol en terre nue ont plus de $50 \%$ de risque supplémentaire de mourir avant l'âge de cinq ans. Cependant, le risque différentiel entre le ciment et la terre nue est statistiquement non significatif. Comme on peut le constater dans le tableau TAB.1 (Voir Appendice), la mortalité des enfants est plus affectée par le materriau de sol entre $1-4$ ans revolus que losqu'ils ont moins d'un an.

La disponibilité en eau/électricité influence globalement la mortalité des enfants de moins de cinq ans. Cette variable impacte de façon significative sur l'âge au décès des enfants de moins d'un an plutôt que sur celui des enfants de $1-4$ ans révolus.

Le rang de naissance contribue significativement à la mortalité infanto-juvénile, aussi bien pour les enfants de moins d'un an que pour ceux de $1-4$ ans révolus. Dans tous les cas, les naissances de rang cinq et plus sont associées à un risque de décès plus important que les naissances des premiers rangs.

L'âge de la mère à l'accouchement agit significativement sur l'âge au décès des enfants de moins de cinq ans. Les enfants nés des mères adolescentes courent $30 \%$ plus de risques de décéder avant leur cinquième anniversaire que ceux des mères âgées de $20-49$ ans. Cette influence serait plus le résultat de la mortalité infantile que de la mortalité juvénile. En effet, les résultats montrent que lorsque l'enfant a déjà atteint un an, l'âge au décès n'est plus associé à l'âge de la mère au moment de l'accouchement, toutes choses égales par ailleurs.

Quel que soit le type de mortalité considéré (infantile et juvenile), les naissances multiples sont associées à un risque de mortalité plus élevé que les naissances simples. En ces termes, comparés aux naissances multiples, les enfants de $0-4$ ans révolus issus des naissances simples ont $30 \%$ de plus de chances de survivre jusqu'à leur cinquième anniversaire.

Le nombre d'enfants de moins cinq ans dans le ménage agit significativement sur l'âge au décès des enfants de moins de cinq ans. Plus ce nombre augmente, plus les chances de survie des enfants diminuent. 
Ce résultat pourrait résulter de la difficile surveillance des enfants, particulièrement turbulants à ces âges, des difficultés financières des parents à assurer simultanément les soins de qualité aux enfants malades et de l'environnement immédiat, particulièrement malsain en milieu urbain ${ }^{20}$.

Le faible poids à la naissance influence négativement la survie des enfants. Plus le poids à la naissace est élevé plus l'enfant a moins de risque de décéder avant le cinquième anniversaire. Il convient de noter que l'influence du poids à la naissance sur la mortalité des enfants se poursuit même après que celui-ci a atteint un an et de façon significative, toutes choses égales par ailleurs.

\section{Conclusion}

L'objectif de ce papier était de tester l'influence des facteurs sanitaires, environnementaux, socio-économiques et culturels, sur l'âge au décès des enfants de moins de cinq ans en recourant aux méthodes statistiques d'analyse des durées.

$\mathrm{Au}$ niveau descriptif, l'analyse de la fonction de survie par la méthode de Kaplan-Meier à permis d'étudier la vitesse d'occurrence des décès (la cinétique des évènements). Cette analyse a permis de conclure à l'existence de deux phases dans l'évolution des enfants de moins de cinq ans selon l'âge : une baisse régulière et rapide de la probabilité de survie avant l'âge de deux ans, puis commence une phase de lente baisse jusqu'au cinquième anniversaire.

L'application du modèle semi paramétrique de Cox sur l'âge au décès des enfants de moins de cinq ans a permis d'identifier les facteurs de mortalité intervenant à chaque période (infantile, juvénile et infanto-juvénile) avant cinq ans.

Dans la période infantile, la mortalité est fortement influencée par le poids à la naissance, le type de naissance et le rang de naissance qui sont des caractéristiques propres à l'enfant. Les deux variables caractéristiques de la mère retenues dans le modèle (le niveau d'instruction et l'âge à l'accouchement) sont des facteurs de mortalité agissant en second plan par rapport aux facteurs propres à l'enfant. Au niveau du ménage, le nombre d'enfants de moins de cinq ans est le plus important facteur de mortalité parmi les autres que sont le niveau de vie et la disponibilité de l'eau/électricité. On notera que le type de sol s'est avéré non significatif à ce stade de la vie de l'enfant. Cela pourrait s'expliquer par le fait qu'à ces âges, les enfants n'ont pas encore un contact permanent avec le sol.

Dans la période juvénile, les plus importants facteurs de mortalité à surveiller sont le type de sol, le nombre d'enfants de moins de cinq ans dans le ménage (caractéristique du ménage) et le poids à la naissance (caractéristique de l'enfant). Les autres facteurs de mortalité juvénile sont le rang et le type de naissance. Le niveau de vie du ménage s'est avéré non significatif sur la mortalité juvénile au Congo. Dans la littérature récente, l'impact du niveau de vie a souvent été controversé. En effet, même si des auteurs comme Barbieri (1991) ont souligné le lien significatif du niveau de vie du ménage sur la survie des enfants, celui-ci est souvent annihilé par d'autres facteurs socioculturels. Aussi, si pour Filmer et Pritchett (2001) il existe une correspondance entre la classification des ménages basée sur les caractéristiques de l'habitat et celle basée sur les dépenses de consommation des ménages (en Inde). L'estimation du niveau de vie à partir des caractéristiques du ménage présente le plus souvent des limites en ce sens qu'elle ne permet pas de distinguer les « riches » et les « pauvres » au niveau intermédiaire de la distribution de l'indicateur (Kobiane (1999)). De façon globale, la mortalité infanto-juvénile au Congo est influencée aussi bien par les caractéristiques inhérentes à l'enfant et à la mère que par les caractéristiques du ménage. Signalons que ses résultats corroborent ceux de nos prédécesseurs qui ont traité du sujet. (Rakotondrabe (2004), Barbieri (1991), Beninguisse (1993), Mboko Ibara (2009)). Toutes les variables du modèle se sont avérées significatives aux différents seuils. Parmi les facteurs, on retrouve en premier, le rang de naissance, le poids à la naissance, le type de naissance, l'âge de la mère à l'accouchement, le niveau d'instruction de la mère, le nombre d'enfants de moins de cinq ans dans le ménage et la disponibilité de l'eau de l'électricité dans le ménage. En second lieu, on retrouve le niveau de vie du ménage et le type de sol.

Ainsi, le rang de naissance jouerait un rôle crucial pour la survie de l'enfant. Contrairement aux premiers nés, les enfants de rang élevé bénéficient généralement de soins de moindre qualité, l'attention accordée par la mère aux enfants diminuant considérablement avec le rang de naissance des enfants. Cette diminution pourrait provenir du surcroît de charge occasionné par une famille relativement nombreuse. Les enfants de rang élevé seraient aussi exposés à de haut risque de mortalité en raison de problèmes obstétricaux croissants avec l'augmentation du nombre d'accouchements et de l'âge de leur mère (Banza Baya (1993)).

\section{RÉFÉRENCES}

Agha, S. 2000. The determinants of infant mortlity in Pakistan, Social Science \& Medecine, Vol 51, pp. 199-208

Aly,Y.H., Grabowsky, R. 1990. Education and child mortality in Egypt, World Development, Vol 18, 5, pp. 733-742.

Ambapour, S. 2001. Note sur la mortalité infantile, Document de Travail no 04/2001, BAMSI, Brazzaville. http :/www.cnsee.org

Ambapour, S., Moussana Hylod, A. 2007 Pauvreté et fécondité au Congo, Document de Travail no 14/2007, BAMSI, Brazzaville. http :/www.cnsee.org

Ambapour, S., Moussana Hylod, A. 2008. Pauvreté et santé nutritionnelle de l'enfant au Congo,Document de Travail $n^{\circ}$ 15/2008, BAMSI, Brazzaville.http :/www.cnsee.org

Banza Baya. 1993. Les déterminants de la mortalité des enfants en milieu urbain au Burkina Faso : cas de Bobo Dioulasso, Université de Montréal, Collection de thèses et mémoires $n^{\circ} 35$.

Barbieri, M. 1991. Déterminants de la mortalité des enfants dans le tiers monde, Dossiers du CEPED $n^{\circ} 18$, Paris.

Beaulière, A., Flori, Y-A., Boidin, B. 2007. Pauvreté des conditions de vie et mortalité infanto-juvénile en Haîti, Sciences Sociales et Santé, Vol 26, 2, pp. 39-77.

Beninguisse G. 1993. Approvisionnement en eau et assainissement : effets sur la morbidité et la mortalité des enfants par maladies diarrhéiques, le cas du Cameroun, Mémoire de fin d'études IFORD. Yaoundé.

Breslow, N.E., (1974). Covariate analysis of censored survival data, Biometrics, 30, pp.89-100

Breslow, N.E. 1975. Analysis of survival data under proportional hazards model, International Statistical Review, 43, pp. 45-58.

Byar, D.P. 1982. Analysis of survival data : Cox and Weibull models with covariates, in Statistics in medical research, V. Mike \& K.E Stanley (eds), pp. 365-401, Wiley, N.Y. 
Caldwell, J. 1979. Education as a factor in mortality decline : an examination of Nigerian data, Population Studies, Vol 33, pp. 395-413.

Caselli, G., Vallin, J., Wunsh,G. 2002.Démographie : analyse et synthèse.III. Les déterminants de la mortalité des enfants. INED, Paris.

Casterlin, J.B., Cooksey, E.C., Ismail, A.F. 1989. Household income and child survival in Egypt, Demography, 26, pp. 1535.

CNSEE \& ORC Macro. 2005. Enquête Démographique et de Santé du Congo.

Courgeau, D., Lelièvre, E. 1989. Analyse démographique des biographies, INED, Paris.

Cox, D.R. 1972. Regression models and life-tables (with discussion), Journal of Royal Statistical Society, Series B, 34, pp. 187-220.

Cox, D.R. 1975. Partial likelihood, Biometrika, 62, 2, pp. 269276.

Drosbecke, J-J., Fichet, B., Tassi, P. 1989. Analyse statistique des durées de vie, Economica

Esrey, S.A. 1996. Water, waste and well-being : a multicountry study, American Journal of Epidemology, 143, pp. 608-623.

Filmer, D., Pritchett, L. 2001. Estimating Wealth effects without expenditure data or tears : an application to educational enrolments in States of India.Demography, vol 38,1, pp.115-132.

Franzen, P.D., Hogan, D.P. 1982. The impact of class, education, and health care on infant mortality in a developing society : the case of rural Thailand, Demography, Vol 19, 3, pp. 391-408.

Gourieroux, C. 1989. Econométrie des variables qualitatives, Economica

Hala, A. 2002. The effect of water and sanitation on child mortality in Egypt, Evironmental Economics Unit, Department of Economics, Gothenburg University.

Hill, C., Com-Nougué, C., Kramar, A., Moreau, T., O'Quigley, J., Senossi, R., Chastang, C. 1996. Analyse statistique des données de survie, Flammarion.

Jacoby, H., Wang, L. 2003. Evironmental determinants of child mortality in rural China : a competing risks approach, WashingtonDC : World Bank.

Kalbfleisch, J.D., Prentice, R.L. 1989. The statistical analysis of failure time data, Wiley.

Kiefer, N.M. 1988. Economic duration data and hazard functions, Journal of Economic Literature, XXVI, pp. 646-679.

Klaauw, van der, B., Wang, L. 2003. Child mortality in rural India, World Bank Working paper, WashingtonDC

Kobiane, J.F. 1999, Pauvreté, structure familiale et stratégie éducative à Ouagadougou, in Education familiale et dynamiques démographiques, sous la direction de M. Cosio, R. Marcoux, M. Pilon et A Quesnel, Paris, CICRED, pp 153182.

Le Golf, J.P. 2003. Modélisation des éléments du parcours de vie. Une introduction, Institut Pavie, http :/www2.unil.ch/pavie

Le Golf, J.P., Forney, Y. 2003. Estimations non-paramétrique avec SPSS. Méthode de Kaplan-Meier et méthode actuarielle,Institut Pavie,http :/www2.unil.ch/pavie
Lelièvre, E. 2010. L'approche biographique des trajectoires individuelles, in Droesbeke, J-J., Saporta, G., (Eds), Analyse statistique des données longitudinales, Technip.

Madise, N., Banda, E., Benaya, K. 2003. Infant mortality in Zambia : socioeconomic and demographic correlates, Social Biology, 50, 1-2, pp. 148-166

Manda, S. 1999. Birth intervals, breastfeeding and determinants of childhood mortality in Malawi, Social Science $\varepsilon$ Medicine, Vol 48, pp. 301-312

Martin, L.G., Trussel, J., Salvail, F.R., Shah, N.M. 1983. Covariates of child mortality in the Philippines, Indonesia, and Pakistan : an analysis based on hazard models, Population Studies, Vol 37, pp. 417-432.

Masuy-Stroobant, G. 2002. Théories et schémas explicatifs de la mortalité des enfants, in G. Caselli, J. Vallin, G. Wunsh (eds), Démographie : analyse et synthèse III. Les déterminants de la mortalité des enfants, pp. 421-438, INED, Paris.

Masuy-Stroobant, G. 2002. Les determinants de la santé et de la mortalité infantiles, in G. Caselli, J. Vallin, G. Wunsh (eds), Démographie : analyse et synthèse III. Les déterminants de la mortalité des enfants, pp : 129-144.

Mboko Ibara, S.B., 2009. Influence des conditions d'existence sur la mortalité de moins de 5 ans en Afrique Centrale. Cas du Cameroun et du Congo, Miméo, Brazzaville.

Melligton, N., Cameron, L. 1999. Female education and child mortality in Indonesia, Melbourne, Research Paper 693, The University of Melbourne.

Mosley, W.H., Chen, L.C. 1984. An analytical framwork forthe study of child survival in developing countries, In "Mosley, W.H., Chen, L.C., (eds.), Child survival : strategies for research, Population and Development Review, supplement to volume 10, pp. 24-4.

Mutunga, C.L. 2007. Environmental determinants of child mortality in Kenya, United Nations University, Research Paper $N^{\circ} 2007 / 83$

Planchet, F., Thérond, P. 2006. Modèles de durée. Applications actuarielles, Economica.

Rakotondrabe. 2004. Statut de la femme, prise de décision et santé des enfants à Madagascar, thèse de doctorat, IFORD, Yaoundé.

Ray, J.C. 1988. Données censurées et modèles de durée, Recherche et Applications en Marketing, 3, 2, pp. 77-88.

Ritschard, G., (2004). Estimer un modèle de Cox en temps continu avec SPSS, Université de Genève, Département d'économétrie, http :/www2.unil.ch/pavie

Sastry, N. 1996. Community characteristics, individual household attributes, and child survival in Brazil, Demography, Vol. 33, 2, pp. 211-229

Schultz, T.P. 1984. Studying the impact of household economic and community variables on child mortality. In "Mosley, W.H., Chen, L.C., (eds.), Child survival : strategies for research, Population and Development Review, supplement to volume 10, pp.215-235.

Trussel, J., Hammerslough, C. 1983. A hazards-model analysis of covariates of infant and child mortality in Sri Lanka, Demography, 20, pp. 1-26.

Woldemicael, G. 1998. The effects of water supply and sanitation on chilhood mortality in urban Erytrea, Journal of biosocial Science, 32, pp. 207-227. 


\section{Appendice : Tableaux et Graphiques}

\section{Tableaux}

\begin{tabular}{|c|c|c|c|}
\hline Facteurs de mortalité & $\begin{array}{c}\text { Enfants 0-11 mois } \\
\text { M1 }\end{array}$ & $\begin{array}{c}\text { Enfants 1-4 ans } \\
\text { M2 }\end{array}$ & $\begin{array}{c}\text { Enfants de 0-4 ans } \\
\text { M3 }\end{array}$ \\
\hline Niveau de vie & $* *$ & ns & $* *$ \\
\hline Pauvre & $(\mathrm{R})$ & $(\mathrm{R})$ & $(\mathrm{R})$ \\
\hline Moyen & 1,3 & 1,0 & 0,99 \\
\hline Riche & $0,6^{* * *}$ & 0,7 & $0,64^{* * *}$ \\
\hline Niveau d'instruction & $* *$ & ns & $* * *$ \\
\hline Sans niveau & $(\mathrm{R})$ & $(\mathrm{R})$ & $(\mathrm{R})$ \\
\hline Primaire & $1,5^{* *}$ & 0,77 & 1,2 \\
\hline Secondaire & 1,0 & 0,65 & $0,75^{* * *}$ \\
\hline Supérieur & 0,6 & 0,1 & $0,5 * * *$ \\
\hline Matériau du sol & ns & $* * *$ & $* *$ \\
\hline Carreaux & 0,76 & $0,2^{* * *}$ & $0,5^{* * *}$ \\
\hline Ciment & 0,94 & 1,0 & 0,85 \\
\hline Terre nue & $(\mathrm{R})$ & $(\mathrm{R})$ & $(\mathrm{R})$ \\
\hline Disponibilité en eau/électricité & $* *$ & ns & $* * *$ \\
\hline Eau et électricité & 0,8 & 1,0 & 0,83 \\
\hline Eau seulement & 1,3 & 1,1 & 1,1 \\
\hline Électricité seulement & $1,4^{* *}$ & 1,6 & $1,6^{* *}$ \\
\hline Aucun des deux & $(\mathrm{R})$ & $(\mathrm{R})$ & $(\mathrm{R})$ \\
\hline Rang de naissance & $* * *$ & $* *$ & $* * *$ \\
\hline Rang 1 & $0,51^{* * *}$ & $0,35^{* * *}$ & $0,6^{* * *}$ \\
\hline Rang 2 & 0,91 & 0,64 & 0,81 \\
\hline Rang 3 & 0,95 & 0,89 & 0,86 \\
\hline Rang 4 & 1,0 & 1,2 & 1,1 \\
\hline Rang 5 ou plus & $(\mathrm{R})$ & $(\mathrm{R})$ & $(\mathrm{R})$ \\
\hline Age de la mère à l'accouchement & $* *$ & ns & $* * *$ \\
\hline $15-19$ ans & $(\mathrm{R})$ & $(\mathrm{R})$ & $(\mathrm{R})$ \\
\hline 20 ans ou plus & $0,7^{* *}$ & 1,0 & $0,7^{* * *}$ \\
\hline Type de naissance & $* * *$ & $* *$ & $* * *$ \\
\hline Simple & $0,33^{* * *}$ & $0,4^{* *}$ & $0,3^{* * *}$ \\
\hline Multiple & $(\mathrm{R})$ & $(\mathrm{R})$ & $(\mathrm{R})$ \\
\hline Nbre d'enfts de $0-4$ ans & $* * *$ & $* * *$ & $* * *$ \\
\hline Quatre enfts ou plus & $(\mathrm{R})$ & $(\mathrm{R})$ & $(\mathrm{R})$ \\
\hline Tois enfts & $0,99 * * *$ & $3,3^{* * *}$ & $1,1^{* * *}$ \\
\hline Deux enfts & 0,94 & 1,4 & $0,97 * * *$ \\
\hline Un enft & $0,85^{* * *}$ & 0,9 & 0,8 \\
\hline Poids de l'enfant à la naissance & $* * *$ & $* * *$ & $* * *$ \\
\hline Surpoids & $0,4^{* * *}$ & $0,43^{* * *}$ & $0,4^{* * *}$ \\
\hline Normal & $0,6^{* * *}$ & $0,44^{* * *}$ & $0,53^{* * *}$ \\
\hline Faible poids & ( R) & $(\mathrm{R})$ & $(\mathrm{R})$ \\
\hline Khi-Deux & $408^{* * *}$ & $164^{* * *}$ & $685^{* * *}$ \\
\hline
\end{tabular}

TAB.1 - Les effets nets selon les facteurs de mortalité des enfants

Note $:$ ns $=$ non significatif $;{ }^{*}$ probabilité $(p)$ significative à $10 \% ;{ }^{* *} p$ significative à $5 \%$;

*** $p$ significative à $1 \% ;(\mathrm{R})=$ modalité de référence. 


\section{Graphiques}

Probabilité de survie des enfants avant le cinquième anniversaire

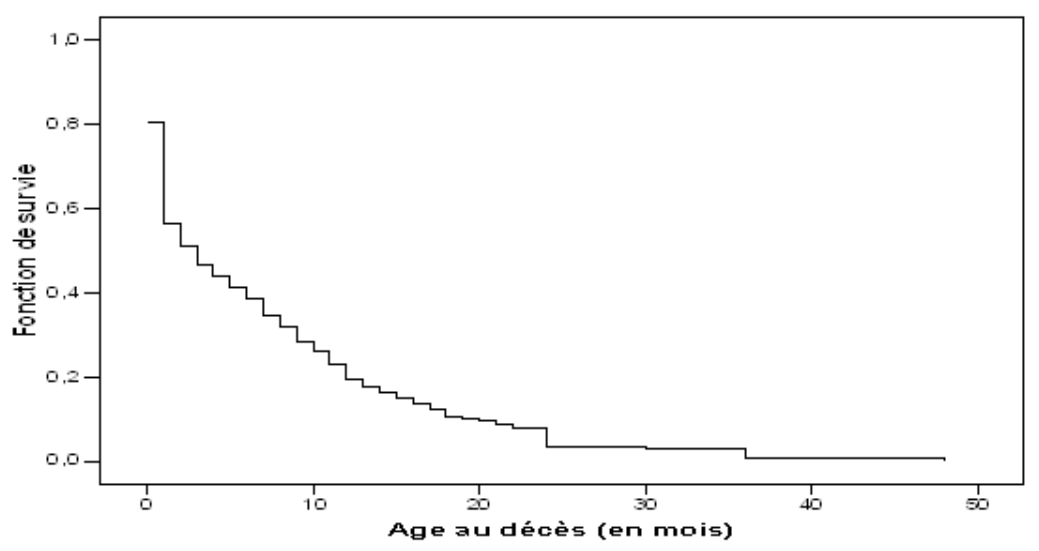

FIG.1 : Estimateur de Kaplan-Meier de la fonction de survie 